\title{
The resistance growing trend of common gram- negative bacteria to the potential antibiotics over three consecutive years: a single center experience in Bangladesh
}

\begin{abstract}
The increasing trend of antibiotic resistance is a global emergence. The susceptibility of the most common gram-negative bacteria (GNB) are reducing day-by-day to potential antibiotics as a result of different enzymatic and non-enzymatic resistancemechanisms of bacteria and disseminating the resistance-gene throughout the intra and inter-bacterial communities rapidly. The objective of this study was to evaluate the resistance developing trends of common GNB against the potential antibiotics over three consecutive years $(2016,2017$ and 2018) in a single center of Bangladesh. The resistance pattern of meropenem, amikacin, ceftazidime and cefepime was fluctuated to Escherichia coli, Klebsiella pneumonia, Acinetobacter baumannii and Pseudomonas aeruginosa in year-to-year pattern and the overall sensitivity reducing trend was observed among those GNB to the selected potential antibiotics. Polymyxin B and colistin was found with relatively higher sensitivity trend to these GNB, but resistance to Klebsiella pneumonia and Acinetobacter baumannii was relatively higher. Ceftazidime was found always with a negative sensitivity trend to Acinetobacter baumannii and Pseudomonas aeruginosa, where others had positive sensitivity trend. Oppositely, amikacin and cefepime was found with highest positive sensitivity trend to $P$. aeruginosa ( 6.0 and 7.0, respectively). These resistance scenarios were found locally, but represented the severity of this crisis worldwide. Resistance developing trend is not limited to the few last resort antibiotics including polymyxin B and colistin. Awareness in antibiotics use, rational use and prescription of antibiotics, restriction of antibiotics in agriculture and livestock etc. are the urgent steps required to tackle this emergence.
\end{abstract}

Keywords: antibiotics, resistance, sensitivity, gram-negative bacteria, emergence
Volume 7 Issue 3 - 2019

\author{
Md. Jahidul Hasan,' Md. Sohrab Hosen, ${ }^{2}$ \\ Sitesh C Bachar ${ }^{3}$ \\ 'Clinical Pharmacy Services, Department of Pharmacy, Square \\ Hospitals Ltd., Bangladesh \\ In-patient Pharmacy Services, Department of Pharmacy, Square \\ Hospitals Ltd., Bangladesh \\ ${ }^{3}$ Department of Pharmacy, University of Dhaka, Bangladesh
}

\begin{abstract}
Correspondence: Md. Jahidul Hasan, Clinical Pharmacist, Clinical Pharmacy Services, Department of Pharmacy, Square Hospitals Ltd., I8/F Bir Uttam Qazi Nuruzzaman Sarak, West Panthapath, Dhaka-1205, Bangladesh, Tel +880191 I0I I 167, Email jahidrj@gmail.com
\end{abstract}

Received: March 26, 2019 | Published: May 09, 2019

\section{Introduction}

The capital Dhaka city of Bangladesh is one of the world's most crowded cities where about 45,000 people live per square $\mathrm{Km}$ area and an estimated 14.4 million people have made this city over populated. ${ }^{1}$ The rapid spread of the infection from one person to another is very common here because of the tropical climate, congested living patterns, lack of active infection control activities in most of the healthcare setups, inadequate pure drinking water supply, lack of proper sewerage and draining system, illiteracy of the peoples and inadequate public as well as healthcare professionals knowledge on occupational hygiene and infection control. ${ }^{2}$ Rate of infections among the hospitalized patients and in the community is very high, and still to date, antibiotics are sold more frequently without having a legal prescription and uses of antibiotics are found irrational in many cases. ${ }^{3}$ Antimicrobial resistance (AMR) is a major concern not only for the local population of Dhaka city but also for the global public health due to the resistance-emergence. Inappropriate use, overuse and unjustified use of antibiotics are considered as the major causes of antibiotics becoming resistant to antibiotics. ${ }^{3,4}$

According to the World Health Organization (WHO), fast developing common bacterial resistance mechanisms to the available antibiotics is a threat for the global human health and day-by-day its severity is increasing, inconceivably. ${ }^{4}$ In Bangladesh, availability of counterfeit antibiotics, incompleteness of individual dosing, injudicious antibiotic prescribing habits of the prescribers and inability of purchase the total prescribed dose of antibiotics are considered as the major key factors for enhancing the rate of antibiotics resistance in the community. ${ }^{3,5}$ Moreover, inappropriate use of antibiotics in the livestock has made this situation more complex nowadays. ${ }^{6}$ In addition, day-by-day the resistance growing trend of the gram-negative bacteria against the few available potential antibiotics is now the major concern and this alarming situation is a vital threat for the global human health. ${ }^{3,6}$ In general, the most popular and potential antibiotics are meropenem, amikacin, ceftazidime, cefepime, polymyxin B and colistin (polymyxin E) ${ }^{7,8}$ These antibiotics are mostly considered as the potential antibiotics when different types of infections are mostly caused by the most common gram-negative bacteria (GNB) including Escherichia coli, Klebsiella pneumonia, Acinetobacter baumannii and Pseudomonas aeruginosa. ${ }^{8}$ The main objective of this study was to observe the resistance developing trend of these common gramnegative bacteria against the few commonly used potential antibiotics over the recent 3 consecutive years in a single hospital setup. 


\section{Material and methods}

This qualitative, retrospective, observational study was conducted in the Square Hospitals Ltd., a tertiary level private hospital in the Dhaka city of Bangladesh. The hospital had a well organized microbiology laboratory. All the microbiological tests of the admitted and out-door patients were performed in that microbiology lab. For microbiological culture sensitivity analysis, the testing methodology was broth dilution and automated analyzer (BD Phoenix ${ }^{\mathrm{TM}}$ M50 Automated Microbiology System) was used for antibiotics' susceptibility analysis and to determine the minimum inhibitory concentration (MIC) of antibiotics in serum. That automated analyzer was validated with the standards of Clinical and Laboratory Standards Institute (CLSI), United States of America. According to the internally established protocol, the microbiology lab kept the records of all types of antibiotics susceptibility patterns against all the tested microorganisms regularly. On the basis of the recorded data, annually an antibiogram report was generated by that lab and disseminated among the hospital clinicians. All the retrospective data on antibiotic susceptibility of clinically relevant gram-negative bacteria were collected from those annually published antibiograms and for the study purpose, antibiograms of the years-2016, 2017 and 2018 were considered. A written approval was taken from the legal authority of the microbiology lab to conduct this study and another written approval was collected from the hospital authority for this study purpose. The ethical approval for this study was taken from the ethical committee of the Square hospital on December, 2018.

\section{Data extraction process and statistical analysis}

The microbiology department recorded year to year all the available antibiotics' susceptibility data including meropenem, amikacin, ceftazidime, cefepime, polymyxin B and colistin against the most common GNB (Escherichia coli, Klebsiella pneumonia, Acinetobacter baumannii and Pseudomonas aeruginosa), and mentioned accordingly in the antibiogram reports annually. The time series analysis technique was used to analyze the selected antibiotics' sensitivity trends on year-to-year (2016-2018) basis to the GNB The antibiotic sensitivity patterns to the GNB were estimated in the antibiogram report of 2016 on 1748, 808, 292 and 431 patients; in the antibiogram report of 2017 on 1572, 960, 355 and 541 patients; and in the antibiogram report of 2018 on 1600, 942, 279 and 618 patients for E. coli, $K$. pneumonia, A. baumannii and $P$. aeruginosa, respectively. The sensitivity patterns of the common GNB were calculated in percentage (\%). All the retrospective susceptibility data (in \%) were extracted from those antibiograms (2016, 2017 and 2018). The sensitivity pattern of ceftazidime against $E$. coli and $K$. pneumonia was not found and hence, not considered in the corresponding cases. This study considered that antibiotic resistance is inversely proportional to that antibiotic's sensitivity to a particular pathogenic GNB. All the data of this study were analyzed by using IBM Statistical Package for the Social Sciences (SPSS) (version 22) software.

\section{Results}

The sensitivity pattern of meropenem to $E$. coli was increased slightly from $2016(98.0 \%)$ to 2017 (98.2\%) but, reduced in 2018 (96.0), significantly (Figure 1). An incremental trend was found in the year-to-year sensitivity pattern of amikacin against $E$. coli (Figure 1). The sensitivity of cefepime against $E$. coli gradually decreased from
2016 to 2018 , whereas polymyxins (polyxin B and colistin) were found with no change in the sensitivity pattern except polymyxin B in 2017 (sensitivity reduced to $99.0 \%$ and increased in the following year) (Figure 1).

In 2017 , the sensitivity patterns of meropenem and amikacin against $K$. pneumonia were increased significantly (meropenem: $64.0 \%$ to $70.0 \%$; amikacin: $61.0 \%$ to $63.7 \%$ ) but, in the next year reduced, remarkably (Figure 2). Cefepime was found with similar pattern of sensitivity in 2016 and $2018(33.0 \%)$ but, significantly increased in 2017 (35.5\%) (Figure 2). A remarkable fluctuation in the sensitivity patterns of polymyxin B was observed from year-toyear and a significant reduction (2016 and 2017: $98.0 \%$ to $94.0 \%$ : 2018 ) was found in colistin's sensitivity pattern against $K$. pneumonia (Figure 2).

In against of $A$. baumannii, an incremental change was observed in the sensitivity patterns of meropenem and a significant reduction was found in case of amikacin from the year 2017 to 2018 (Figure 3). The sensitivity of ceftazidime was falling significantly from 2016 to 2018 but, cefepime's sensitivity was increased remarkably in the year 2018 $(11.6 \%$ to $16.0 \%)$ (Figure 3 ). The overall sensitivity of polymyxin B and colistin was reduced significantly from 2016 to 2018 .

Meropenem's sensitivity to $P$. aeruginosa was increased from 2016 to 2017 (61.0\% to 65.4\%) but, reduced in 2018 (63.0\%) (Figure 4). The sensitivity of amikacin increased significantly from 2016 to 2018, incrementally whereas, ceftazidime's sensitivity fallen from $64.0 \%$ (2016) to $59.6 \%$ (2018) (Figure 4). With a significant reduction in the sensitivity of cefepime in the year 2017 (57.8\%) but, in 2018, it boosted-up (69.0\%), significantly (Figure 4$)$. In case of polymyxin B and colistin, there was no change in sensitivity observed in 2016 and 2018 but, in 2017, both the antibiotics were found with drastically reduced sensitivity $(89.0 \%$ and $97.0 \%$, respectively) to $P$. aeruginosa (Figure 4).

When the sensitivity pattern of an antibiotic to a specific organism is reduced that means, the organism is more likely resistant to that particular antibiotic. In comparing the antibiotic resistance patterns of the selected antibiotics from the year 2016 to 2018, resistance potentiality of $E$. coli increased $2.0 \%$ more to meropenem, reduced significantly to amikacin and cefepime, and polymyxin B and colistin showed no change in their resistance patterns (Table 1).

There was no change in the resistance patterns of meropenem and cefepime to $K$. pneumonia but, polymyxin B and colistin were found with reduced sensitivity $(1.0 \%$ and $4.0 \%$, respectively) to $K$. pneumonia from the year 2016 to 2018 (Table 1). Positive resistance trend was significantly observed among amikacin, ceftazidime, polymyxin $\mathrm{B}$ and colistin against $A$. baumannii but, negative trend was found in meropenem and cefepime in considering the year 2016 to 2018 (Table 1). In considering the one-step sensitivity-difference from 2016 to 2018, the highest (resistance $>2$ was only considered) resistance developing trend was observed in $K$. pneumonia to colistin (4.0); A. baumannii to ceftazidime (3.8) and colistin (3.0); $P$. aeruginosa to ceftazidime (4.4) (Table 1). With no change in the resistance patterns of polymyxin B and colistin against $E$. coli and $P$. aeruginosa from 2016 to 2018 was observed. A significant increasing resistance pattern was observed in ceftazidime to all corresponding GNB. Except ceftazidime, all other antibiotics had resistance lowering 
trend to P. aeruginosa (Table 1). For some antibiotics, the sensitivity trend was found with significantly positive (only considered sensitivity $>2$ ), such as amikacin to $E$. coli (4.0); meropenem and cefepime to $A$. baumannii (3.0 and 3.0, respectively); amikacin and cefepime to $P$. aeruginosa (6.0 and 7.0, respectively) (the highest sensitivity trends observed in this study) (Table 1).

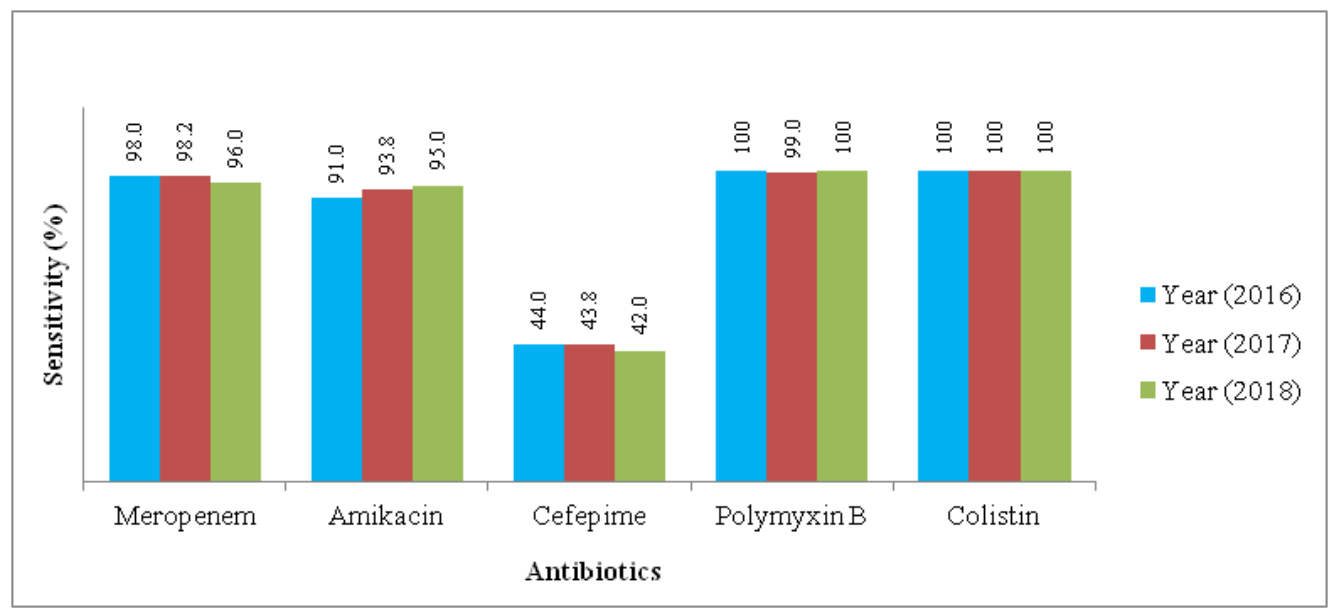

Figure I The antibiotic sensitivity patterns of $E$. coli.

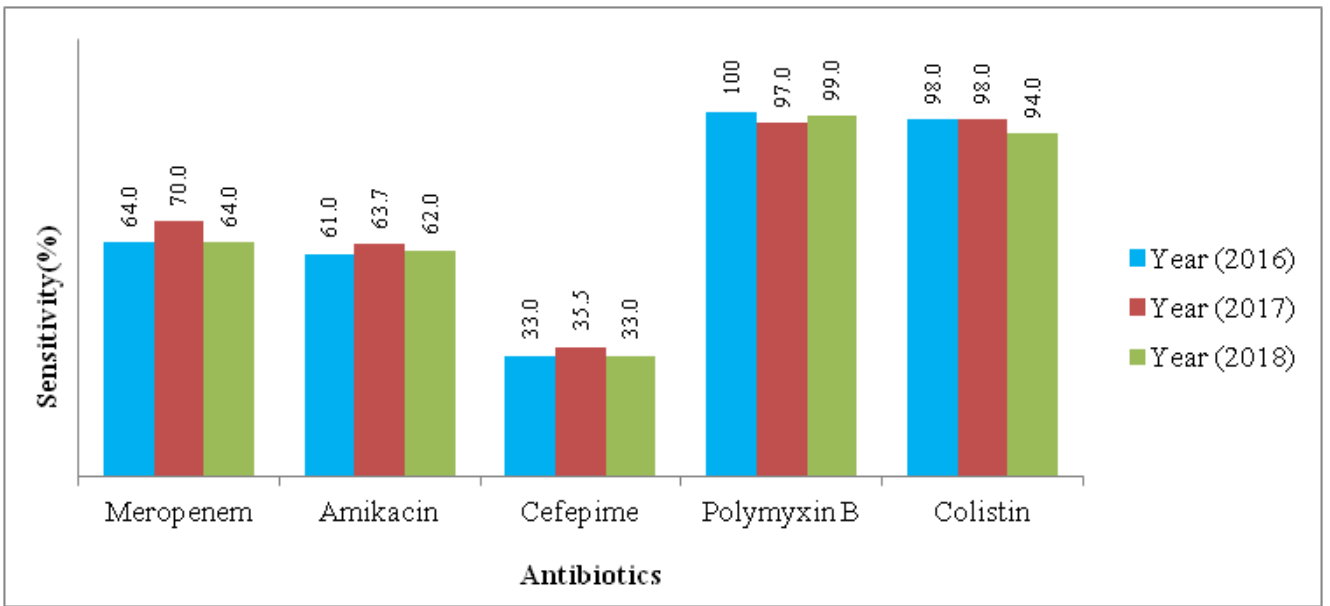

Figure 2 The antibiotic sensitivity patterns of $K$. pneumonia.

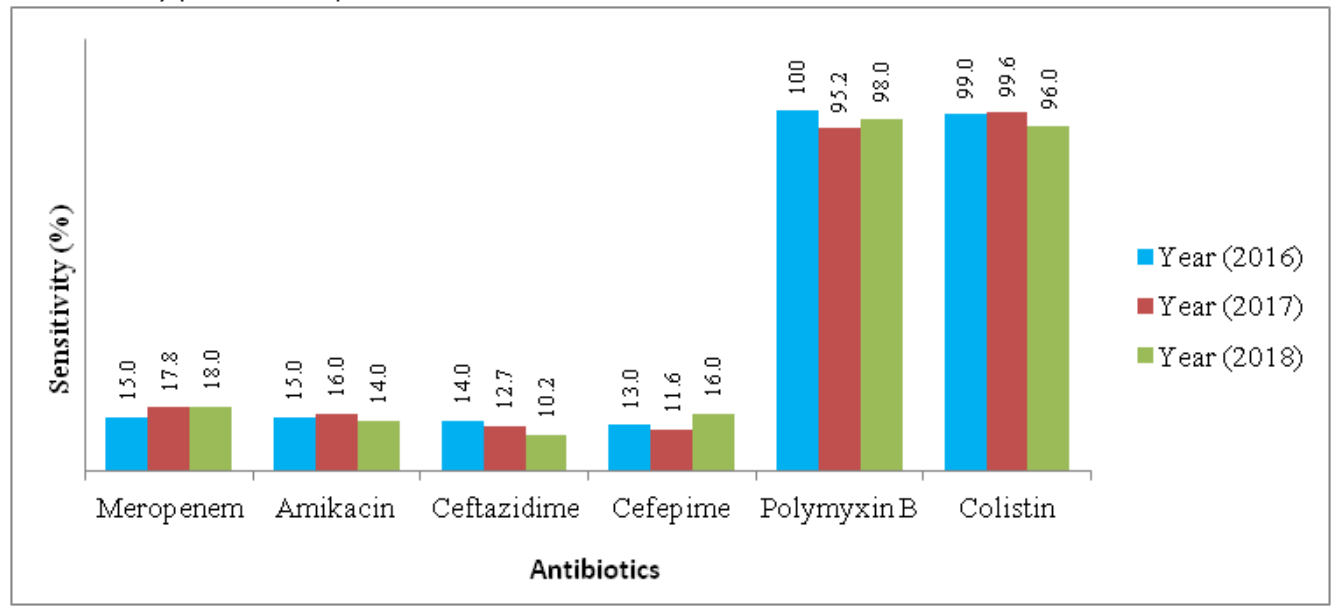

Figure 3 The antibiotic sensitivity patterns of $A$. baumanni. 


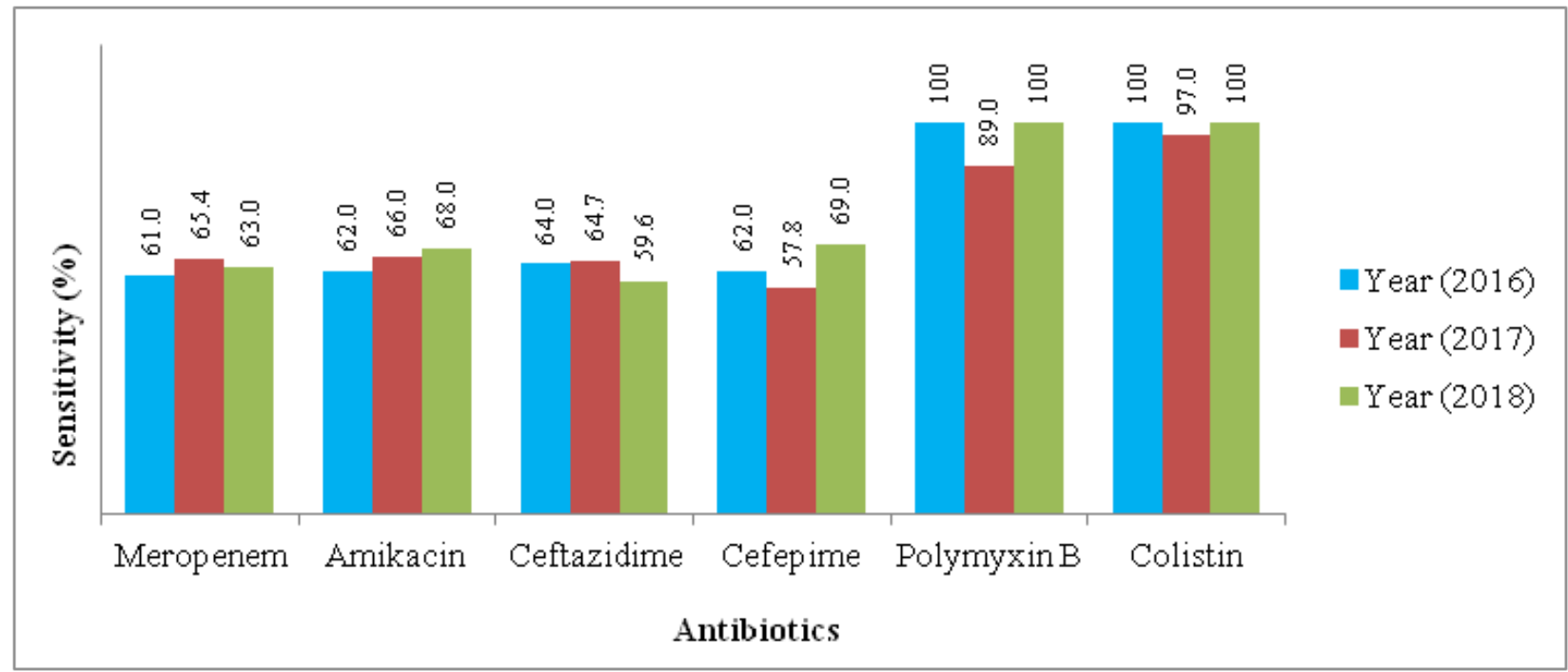

Figure 4 The antibiotic sensitivity patterns of $P$. aeruginosa.

Table I Antibiotic resistance patterns

\begin{tabular}{lllll}
\hline \multirow{2}{*}{ Antibiotics } & \multicolumn{4}{l}{ Antibiotic resistance patterns change from 2016 to $2018(\%)$} \\
\cline { 2 - 5 } & E. coli & K. pneumonia & A. baumannii & P. aeruginosa \\
\hline Meropenem & $+(2.0)$ & No change & $-(3.0)$ & $-(2.0)$ \\
Amikacin & $-(4.0)$ & $-(1.0)$ & $+(1.0)$ & $-(6.0)$ \\
Ceftazidime & - & - & $+(3.8)$ & $+(4.4)$ \\
Cefepime & $-(2.0)$ & No change & $-(3.0)$ & $-(7.0)$ \\
Polymyxin B & No change & $+(1.0)$ & $+(2.0)$ & No change \\
Colistin & No change & $+(4.0)$ & $+(3.0)$ & No change \\
\hline
\end{tabular}

'+', positive trend in resistance development; '-', negative trend in resistance development

\section{Discussion}

In this study, the resistance patterns of antibiotics changed yearto-year, individual potential antibiotic was found with different sensitivity to different GNB in different years and polymyxin B and colistin was the highest sensitive antibiotics to all 4 types of GNB in every individual year. The antibiotic resistance trend among the common GNB has reached in the boarder-line of the crisis and irrational use of antibiotics has been considered as the major contributor to this emergence. ${ }^{9,10}$ In Bangladesh, the injudicious use of antibiotics is a common tendency in all classes of peoples including healthcare professionals also. ${ }^{11}$ When an antibiotic is used improperly, then the corresponding bacterium became resistant to that antibiotic by developing resistant-gene and finally, this resistantgene is disseminated in the population in as a matter of time., ${ }^{3,12}$ This increasing trend of resistant organisms causes more infectionassociated hospitalization, treatment failure, mortality and treatment cost. ${ }^{8,13}$ In this study, the sensitivity patterns of meropenem, amikacin, ceftazidime and cefepime were significantly lower (all were found below $50 \%$ of the sensitivity level) than last resort polymyxin B and colistin to A. baumannii in every selected year. Both polymyxin B and colistin are under the polymyxins group and this is considered as one of the last resort antibiotic groups, globally. ${ }^{8}$ Tigecycline is another last resort antibiotic ${ }^{14}$ but, the use of tigecycline is very limited by the prescribers with limited sensitivity data.

Study found an increased susceptibility of $E$. coli to cefixime in one hospital but, a decreased susceptibility was found in another hospital of the same city at the same time in Bangladesh. ${ }^{3}$ The major reasons behind this incidence is not completely clear to date. Horizontally rapid resistance-gene transfer among the same or different bacterial species, species to species inter-transmission of resistance-genetic materials, amplification of the resistance-gene in the genome ${ }^{15}$ and other unknown mechanisms are the main contributor in the variable susceptibilities of organisms to corresponding antibiotics. ${ }^{3}$ The genome-wide repeating tendency of bacteria with developing pseudogenes is very limited in comparison to carry a precise genome. ${ }^{16}$ "Deletional bias" is a process where bacteria rapidly miss out genes in absence of any selective pressure. ${ }^{3,15,17}$ The expression of antibioticinactivating enzymes (such as Beta-lactamase and chromosomeencoded AmpC cephalosporinase) and non-enzymatic mechanisms. ${ }^{18}$ In Bangladesh, the insignificant use of antibiotics in the agricultural 
sector and antibiotics use can lead to transitional selective pressure. As a result, expectation to rapid changes in antibiotic susceptibility is developed. ${ }^{3}$ In the present study, the trend of developing antibiotic resistance among the most common GNB was found with frequent ups and downs (except the susceptibility of colistin to E. coli in 2016, 2017 and 2018) in year-to-year pattern at the same hospital. Study found that pathogenic bacteria can be more resistant to the most common antibiotics within a relatively short time. ${ }^{3}$

The resistance developing trend of GNB is remarkable and very challenging to defense even with the last line potential antibiotics. ${ }^{19}$ According to multiple studies, GNB are responsible for $45-70 \%$ and $20-30 \%$ of ventilator-associated pneumonia (VAP) ${ }^{20}$ and catheterrelated bloodstream infections, ${ }^{21}$ respectively; and also cause of ICUacquired sepsis, surgical site and urinary tract infections (UTI). ${ }^{19}$ The degree of resistance developed by a particular GNB is related to the number of antibiotics is inactive to that organism. If the GNB is non-susceptible to at least one agent among three or more antibiotic groups, then it is called Multidrug-resistant (MDR) organism; if it is non-susceptible to at least one of all antibiotic groups, but two or fewer antibiotic groups, then it is called extensively drug-resistant (XDR) organism and if it is non-susceptible to all agents in all antibiotic groups, then it is called pandrug-resistant organism. ${ }^{22}$ Chromosomal genetic-mutation in the GNB is one of the major mechanisms that can increase the expression of intrinsic resistance mechanism and the severity of potential antibiotic resistance is close related to this intrinsic mechanism. ${ }^{23}$ In our study, the year-to-year reducing susceptibility of the selected GNB to most of the potential antibiotics including meropenem, ceftazidime, polymyxin B and colistin is a huge threat not only for the local population, but also for the global human health. Globally, the crisis of new potential antibiotics in the market-pipeline and rapidly increasing trend of antibiotic resistance will welcome the newer approach "practical not perfect". According to this proposed strategy, antibiotics with increased level of resistance will be withdrawn from the market completely and after a period of time when the antibiotic will have uniform effectiveness, then it will come back again. ${ }^{24}$ Nowadays, the challenge against this antibiotic resistance is referred to as "the silent tsunami facing modern medicine". ${ }^{25}$ Now, it is the topic in numerous international health conferences and summits, and is one of the most juicy topics in different scientific researches, guidelines and recommendations. ${ }^{26}$ Our single center experience shows an up-coming very challenging future against the resistant GNB-induced infections and rationalism in the antibiotics uses is highly required at this moment to defeat this resistance-trend. Global awareness and involvement of mass-media has no alternate to overcome this major human health-crisis.

\section{Conclusion}

The rapidly increasing trend of antibiotic resistance in the most common GNB is an alarming issue, globally. In Bangladesh, this scenario is same and this study found a year-to-year variable nature in the resistance patterns of common GNB to the most potential antibiotics and a reducing sensitivity trend was found in most of the antibiotics. To effectively tackle this global crisis, awareness on antibiotic uses and rational antibiotic practice is mostly required at this moment.

\section{Acknowledgments}

All authors of this study are very grateful to all doctors and authority of Square hospital because of their permission for this study and all kind of cooperation as well as time to time supports during the study.

\section{Conflicts of interest}

Authors declare that there is no conflict of interest.

\section{References}

1. Rahman M. An urban reader. Dhaka: University Press; 2016.

2. Central Intelligence Agency. The CIA World Fact Book. New York: Skyhorse Publishing; 2017.

3. Fariba MH, Sami AB, Bhattacharjee D, et al. Change in Antibiotic Susceptibility Pattern of Clinical Bacterial Isolates from Two Hospitals in Dhaka, Bangladesh over a Period of Three Years. Microbiol Res J Int. 2017;22(6):1-10.

4. Hashemi S, Nasrollah A, Rajabi M. Irrational antibiotic prescribing: a local issue or global concern? EXCLI J. 2013;12:384-395.

5. Islam MS. Therapeutic drug use in Bangladesh: Policy versus practices. Indian J Med Ethics. 2013;10(3):24-25.

6. Akond MA, Alam S, Hassan SMR, et al. Antibiotic resistance of Escherichia coli isolated from poultry and poultry environment of Bangladesh. Am J Environ Sci. 2009;5(1):47-52.

7. Hawkey PM, Warren RE, Livermore DM, et al. Treatment of infections caused by multidrug-resistant Gram-negative bacteria: report of the British Society for Antimicrobial Chemotherapy/Healthcare Infection Society/British Infection Association Joint Working Party. J Antimicrob Chemother. 2018;73(suppl_3):iii2-iii78.

8. Hasan MJ. Polymyxins Nebulization over Intravenous Injection: Pharmacokinetics and Pharmacodynamics-Based Therapeutic Evaluation. $J$ Pharm Res Int. 2018;25(4):1-10.

9. Rossolini GM, Arena F, Pecile P, et al. Update on the antibiotic resistance crisis. Curr Opin Pharmacol. 2014;18:56-60.

10. Martens E, Demain AL. The antibiotic resistance crisis, with a focus on the United States. J Antibiot. 2017;70(5):520-526.

11. Rahman MS, Huda S. Antimicrobial resistance and related issues: An overview of Bangladesh situation. Bangladesh $J$ Pharmacol. 2014;9(2):218-224.

12. Davies J, Davies D. Origins and evolution of antibiotic resistance. Microbiol Mol Biol Rev. 2010;74(3):417-433.

13. Ventola CL. The antibiotic resistance crisis, Part 1: Causes and threats. Pharm Ther. 2015;40(4):277-283.

14. Hasan MJ, Rabbani R, Bachar SC, et al. High Dose Tigecycline-Induced Mitochondrial Dysfunction-Associated Acute Metabolic Acidosis: A Retrospective Study. J Mol Genet Med. 2019;13(1):407.

15. Laehnemann D, Peña-Miller R, Rosenstiel P, et al. Genomics of rapid adaptation to antibiotics: Convergent evolution and scalable sequence amplification. Genome Biol Evol. 2014;6(6):1287-1301.

16. Kuo CH, Moran NA, Ochman H. The consequences of genetic drift for bacterial genome complexity. Genomes Res. 2009;19(8):1450-1454.

17. Mira A, Ochman H, Moran NA. Deletional bias and the evolution of bacterial genomes. Trends Genet. 2001;17(10):589-596.

18. Alekshun MN, Levy SB. Molecular mechanisms of antibacterial multidrug resistance. Cell. 2007;128(6):1037-1050.

19. Ruppé É, Woerther P, Barbier F. Mechanisms of antimicrobial resistance in Gram-negative bacilli. Ann Intensive Care. 2015; 5(1):61. 
20. Barbier F, Andremont A, Wolff M, et al. Hospital-acquired pneumonia and ventilator-associated pneumonia: recent advances in epidemiology and managment. Curr Opin Pulm Med. 2013;19(3):216-228.

21. Surveillance of nosocomial infections in critically ill adult patients. France: REA-Raisin Network; 2012.

22. Magiorakos AP, Srinivasan A, Carey RB, et al. Multidrug-resistant, extensively drug-resistant and pandrug-resistant bacteria: an international expert proposal for interim standard definitions for acquired resistance. Clin Microbiol Infect. 2012;18(3):268-281.

23. Ruppé É, Woerther PL, Barbier F. Mechanisms of antimicrobial resistance in Gram-negative bacilli. Ann Intensive Care. 2015;5(1):61.
24. Chennai Declaration Team. Chennai declaration: 5-year plan to tackle the challenge of anti-microbial resistance. Indian J Med Micro. 2014;32(3):221-228.

25. Cox D. Antibiotic resistance: the race to stop the silent tsunami facing modern medicine. The Guardian; 2015.

26. Exner M, Bhattacharya S, Christiansen B, et al. Antibiotic resistance: What is so special about multidrug-resistant Gram-negative bacteria? GMS Hyg Infect Control. 2017;12:Doc05. 